\title{
Bacillus polymyxa
}

National Cancer Institute

\section{Source}

National Cancer Institute. Bacillus polymyxa. NCI Thesaurus. Code C86166.

A species of facultatively anaerobic, Gram-positive, rod shaped bacteria assigned to the phylum Firmicutes. This species is spore forming, catalase positive, oxidase negative, indole negative, does not produce hydrogen sulfide, reduces nitrate, and hydrolyzes starch and esculin. B. polymyxa, also known as Paenibacillus polymyxa, is found in the rhizospheres of plants and is used to produce antimicrobial compounds and to enhance the growth of plants. 